\title{
RAMAN SCATTERING PHOTONIC AND POLARITONIC RESONANCES IN SEMICONDUCTOR MICROCAVITIES
}

\author{
B. Jusserand and A. Fainstein \\ CNET/Laboratoire de Bagneux, BP107, 92225 Bagneux Cedex, France
}

\begin{abstract}
Single and double optical resonant Raman scattering is demonstrated in semiconductor microcavities. Its evolution towards excitonic ingoing resonance relies on cavity-polaritons as intermediated steps of the inelastic scattering. Implications for high-sensitivity spectroscopy and low-power stimulated processes are emphasized.
\end{abstract}

PACS numbers: $63.20 .-\mathrm{e}, 82.80 . \mathrm{Ch}$

\section{Introduction}

Photon confinement in wavelength-scale optical resonators deeply modifies light-matter interaction processes such as absorption or spontaneous emission taking place inside the cavity. In this paper, we show that optical resonances also modify higher order processes, namely Raman scattering by phonons in planar semiconductor microcavities. Depending on the experimental configurations, ingoing and outgoing photons can be independently or simultaneously tuned to the photonic quantized levels of the cavity, leading to large enhancement of the light scattering external cross-section and directionality. Up to five orders of magnitude enhancement can be achieved in the double optical resonance (DOR) configuration [1].

Going beyond these modifications due to purely photonic effects, we report on the additional enhancement which appears when the ingoing photon energy approaches the cavity polaritons associated to the strong coupling between confined photons and excitons of quantum wells embedded at the center of the cavity [2]. In a high finesse sample, exhibiting a vacuum Rabi splitting of $4.5 \mathrm{meV}$, the external cross-section increases by 250 when going from pure DOR to cavity polariton mediated scattering (CPMS), a factor comparable to excitonic enhancement measured on Raman scattering in bare quantum wells. On the other hand, we do not observe any Rabi splitting in the CPMS resonance curve, which we assign to the dominant contribution of uncoupled localized excitons, coexisting with cavity polaritons. However, we demonstrate a significant decrease in the ingoing directionality as compared to the DOR case. Unlike many experimental conseyuences of the strong coupling, this result cannot be explained in a purely photonic approach (linear dispersion) but requires cavity polariton mediation. 


\section{Double optical resonance}

It is well established from a second-order perturbation theory that the Raman cross-section writes

$$
\sigma_{1} \propto\left|\sum_{i, j} \frac{\left\langle 0\left|H_{\mathrm{MR}}\right| j\right\rangle\left\langle j\left|H_{\mathrm{e}-\mathrm{ph}}\right| i\right\rangle\left\langle i\left|H_{\mathrm{MR}}\right| 0\right\rangle}{\left(E_{\mathrm{L}}-E_{i}\right)\left(E_{\mathrm{L}}-E_{j}\right)}\right|^{2},
$$

where $H_{\mathrm{MR}}$ and $H_{\mathrm{e}-\mathrm{ph}}$ describe the exciton-photon and exciton-phonon interaction, respectively, and $E_{\mathrm{L}}$ is the laser energy. Because of the resonant denominators in Eq. (1), $\sigma_{1}$ displays incoming $\left(E_{\mathrm{L}}=E_{i}\right)$ and outgoing $\left(E_{\mathrm{L}}=E_{j}\right)$ resonances on intermediate states $|i\rangle(|j\rangle)$, either uncoupled electron-hole pairs or exciton states, with energies $E_{i}\left(E_{j}\right)$. The cross-section contains two squared matrix elements of light-matter interaction, due to the incoming and outgoing photon vertexes. These matrix elements are proportional to the electric field amplitude of the laser and the Stokes photon, respectively. Consequently, if these amplitudes are enhanced in a cavity, the Raman scattering efficiency should be fourth-order in the enhancement factor.

Contrary to luminescence experiments in microcavities which are usually performed by excitation above the structure stop-band, the excitation energy is not a free parameter in a Raman experiment: $t w o$ photons with energies differing by a fixed amount (given by the excitation under study) must get into, or go out from, the structure. A double resonance condition can still be met by exploiting the continuum of in-plane optical modes. In fact, if a photon propagating along the cavity axis resonates at an energy $\omega_{\mathrm{s}}$, one incident at an angle $\theta_{0}$ (measured in air) will resonate at a larger energy given approximately by

$$
\omega_{\mathrm{i}}=\omega_{\mathrm{s}} / \cos \left(\theta_{0} / n_{\text {eff }}\right)
$$

provided that an effective index $n_{\text {eff }}$ is used to transform external angles into internal ones. Because of the large penetration of the resonant field inside the Bragg mirrors, this index does not equal the cavity one but takes some intermediate value. When $\left(\omega_{i}-\omega_{s}\right)$ is tuned to an excitation of the system, the conditions for the observation of optical double resonant Raman scattering are obtained.

We have reported recently such a large enhancement [1] in a structure consisting of an AlAs half-wave spacer enclosed by GaAs/AlAs DOR's (20 pairs below, 13.5 above) with two $12 \mathrm{~nm}$ wide $\operatorname{In}_{0.14} \mathrm{Ga}_{0.86}$ As quantum wells (QW's) located at its center. A finesse of $\approx 300$ was estimated for this structure from reflectivity measurements. The sample was grown by molecular beam epitaxy on a GaAs wafer and the growth conditions were such that the thickness of all layers varies with position enabling the tuning of the cavity mode by displacing the spot under consideration.

Raman spectra taken on this sample are presented in Fig. 1 , for $\theta_{0}=54^{\circ}$ with crossed (allowed) polarizations and at an excitation energy of $1.35 \mathrm{eV}$, below the QW's gap to avoid the QW's luminescence. For the bottom spectrum, the angle of incidence and the spot under observation in the cavity sample correspond to a double-resonant condition tuned to the energy of the bulk-GaAs longitudinal optical (LO) phonon, seen as the largest peak in Fig. $1\left(\approx 295 \mathrm{~cm}^{-1}\right)$. A spectrum 


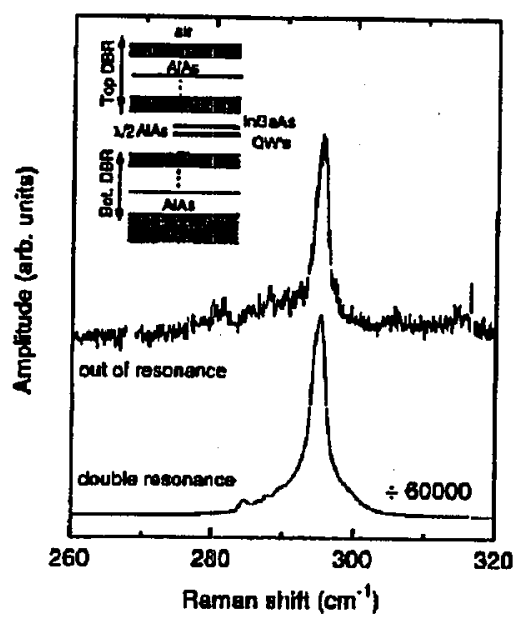

Fig. 1. Raman spectra taken in crossed configuration on the full cavity sample with a spot position corresponding to double optical resonance and out of resonance conditions, respectively.

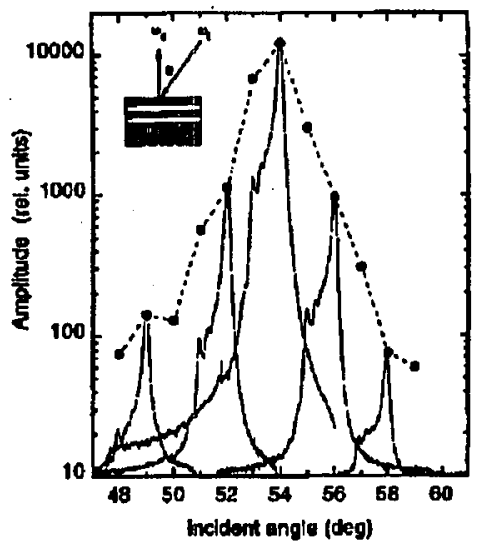

Fig. 2. Dependence of the Raman scattering amplitude (full dots) as a function of $\theta_{0}$, for the cavity tuned along $z$ to the LO-peak. The dashed line is only a guide to the eye. Raman spectra, compressed in the horizontal scale and shifted so that the larger peak coincides with the respective incidence angle, are also shown for illustration.

taken from the same sample but completely detuned by displacing the spot under observation, is also shown. Almost five orders of magnitude difference in the Raman intensity is observed.

The Raman scattering enhancement in a planar microcavity is critically sensitive on both incidence and collection angle, as deduced from Eq. (2). In Fig. 2, we present the amplitude of the largest Raman peak in Fig. 1 as a function of the angle of incidence $\theta_{0}$, all the other parameters being kept constant. More than two orders of magnitude variations of the Raman amplitude are observed by ro- 
tating the incidence angle less than $4^{\circ}$, thus going from double to single resonance (the Stokes photon is always kept in resonance). The angular dependence HWHM of the order of $1^{\circ}$ is consistent, according to Eq. (2), with the reflectivity peak HWHM measured for the same structure $(\approx 0.3 \mathrm{~nm})$. Similar results have been also observed for the collection angle. In fact, such small ingoing and outgoing acceptances are likely to limit the observed enhancement with respect to the maximum calculated one, because of the finite apertures used in the Raman setup.

\section{Quantitative model for the Raman efficiency}

To give a quantitative estimate of the Raman cross-section in microcavities, a model of the eigenvectors of the phonons involved in the Raman process would be needed. Moreover, as the photon field decays slowly in the Bragg mirrors, this modelling should include the vibrations in the whole sample. A detailed analysis of these vibrations has been reported recently [3]. However, to emphasize the significant information on the cavity effect, it is more convenient to assume a vibrational amplitude constant over the cavity and vanishing outside. The cross-section is then approximated as the product of the internal field intensity (at the laser frequency and for a chosen incidence angle) and the radiation intensity (at the fixed Stokes frequency and emission angle), both integrated throughout the cavity length. Using this approach, one recovers qualitatively the fourth power dependence on the field amplitude which we introduced previously. Moreover, both internal intensity and radiation distribution are easily obtained along the lines of this standard electromagnetic theory of layered media.

To improve the validity of this approach, better adapted samples have been designed in which the cavity exhibits specific phonons [4]. It is made of a GaAs/AlAs superlattice, filling up the whole cavity, enclosed with GaAlAs/AlAs Bragg reflectors. Both GaAs-like LO phonons and folded acoustic phonons in the superlattice appear at frequencies outside of the vibrational range of the reflectors. In order to collect a large set of experimental data and to compare the relative intensities to the model, two different samples have been studied with and without the top Bragg reflector. The second sample (half-cavity) exhibits a weaker, but still significant, enhancement of the Raman cross-section as well as a larger angular acceptance. Moreover, for both samples, double resonance experiments, obtained in quasi-backscattering, have been compared to single resonance ones. In the latter case, the Stokes photons are collected at the edge of the layer, thanks to the guiding property of the cavity structure in these new samples, while the excitation is done along the cavity axis. Hence, only the incident photon is enhanced by cavity confinement.

The comparison between measured and calculated Raman intensities is shown in Fig. 3 for the GaAs-like LO phonon in the superlattice and in the four differ'ent experimental situations [5]. The only free parameter is the absolute intensity at the maximum resonance for one of the four curves (we have chosen the single resonance on the half-cavity). The overall agreement is quite good, in particular for the half-cavity. For the full cavity, the measured enhancement is smaller than the expected one, even when the finite collection aperture is taken into account, as done here. This residual discrepancy has been attributed to some fluctuations of 


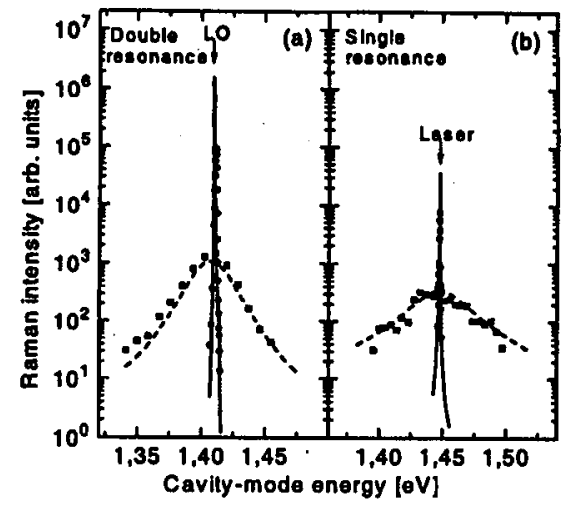

Fig. 3. Comparison between measured and calculated variation around the resonance of the scattering intensity for both single and double resonance conditions on both full (circles) and half (squares) cavity samples.

the layer thicknesses in the real sample, which lower the real finesse with respect to the nominal one, and to distribution of angles due to the slight focussing of the incident beam. The full-cavity structure is much more sensitive to the latter correction because of its smaller angular acceptance. Moreover, the fact that this acceptance decreases with increasing average incidence angle could explain the better agreement obtained in the single resonance experiment, performed at normal incidence. Finally, the finite size of the laser spot on a sample with an intentional gradient of thickness also contributes in reducing the observed enhancement.

Thanks to the high finesse of the optical cavities, one is able to selectively enhance some spectral features in the Raman spectra with respect to the others. Changing the relative energies of the cavity mode and the laser, either by tuning the laser or by moving the sample, one can bring to exact resonance with the cavity mode at normal incidence different outgoing energies corresponding to different vibrations in the sample, while the ingoing photon remains within the cavity resonance at fixed angle. Even stronger selectivity could be obtained by adjusting this angle to achieve double resonance for each Raman shift, at the price of an increased experimental complexity. The rich spectrum of phonons is associated to interface optical vibrations in the structure and a detailed analysis can be found in Ref. [3]. Here, we want to highlight the possibility of extracting some weak lines from complex Raman spectra.

\section{Cavity polariton mediated Raman scattering}

We have demonstrated in the previous part that an optical microcavity confines spatially and spectrally the photon field. When quantum well excitons at energy $E_{x}$ are included in a high finesse planar semiconductor microcavity, a "strong-coupling" regime appears where coherent Rabi oscillations between the exciton and cavity-photon states occur [5]. The polariton wave function may be written as

$$
|K\rangle=\cos \phi|\mathrm{e}, 0\rangle+\sin \phi|\mathrm{g}, 1\rangle
$$


where $|e, 0\rangle(|g, 1\rangle)$ corresponds to one $e-h$ pair in the exciton (ground) state and zero (one) photons. $\tan 2 \phi=-\Omega / \Delta$, where $\Omega$ is the Rabi splitting and $\Delta$ - the exciton-photon detuning. For large detunings the modes retain their original uncoupled character, while a strong mixing and energy splitting (evident in luminescence or reflectivity spectra) develop close to degeneracy. These coupled modes, so-called "cavity polaritons," should have strong consequences also in higher-order optical processes. Proof of the involvement of polaritons as intermediate steps in light scattering has been pursued for a long time. However, while bulk-polariton mediation has been demonstrated in Brillouin scattering experiments [6], the more subtle differences between perturbative (exciton) and polariton theories of first-order resonant Raman scattering in bulk semiconductors have not yet found a clear experimental demonstration [7].
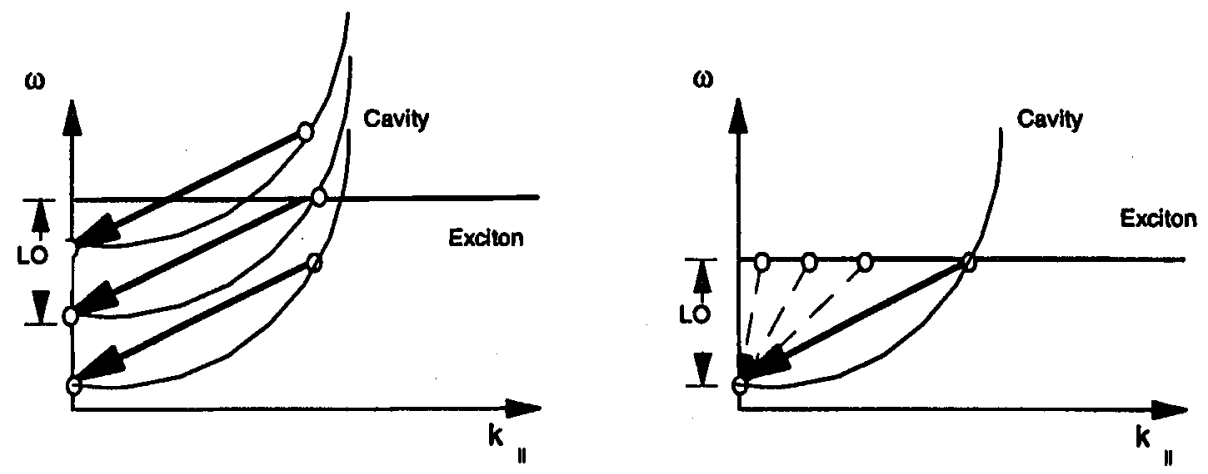

Fig. 4. Schematic representation of the two Raman experiments devoted to the demonstration of cavity-polariton mediation in microcavities: incident energy variation (left) and incident angle variation (right).

In order to probe cavity polariton mediated Raman scattering, we have realized experiments according to the schematic pictures in Fig. 4: starting from a double optical resonance, we have tuned the laser energy and shifted the sample in order to always stay in DOR conditions with the scattered light emitted at normal incidence and to allow the incident energy to cross the excitonic one in the cavity. The experiments were done at $1.7 \mathrm{~K}$ with laser powers ranging from $50 \mu \mathrm{W}$ to $10 \mathrm{~mW}$ (depending on the photon energy). Even with these low temperatures and reduced powers we were not able to separate Raman signals from luminescence under outgoing resonance conditions. In Fig. 5 we present Raman spectra taken according to this experimental procedure, with $\theta_{0}=50^{\circ}$ tuned for DOR at the GaAs-like longitudinal optical phonon ( $\mathrm{LO}=295 \mathrm{~cm}^{-1}$ ). The incoming exciton resonance is close to the spectrum labeled with $*$. In this experiment, we separate the varying excitonic resonance contribution from the optical-like one, which is held fixed to its maximum value.

The laser energy dependence of the Raman intensity, corresponding to the spectra presented in Fig. 5, is also shown in the same figure (full circles). The resonance scan can be nicely followed from pure DOR to CPMS, where exciton 

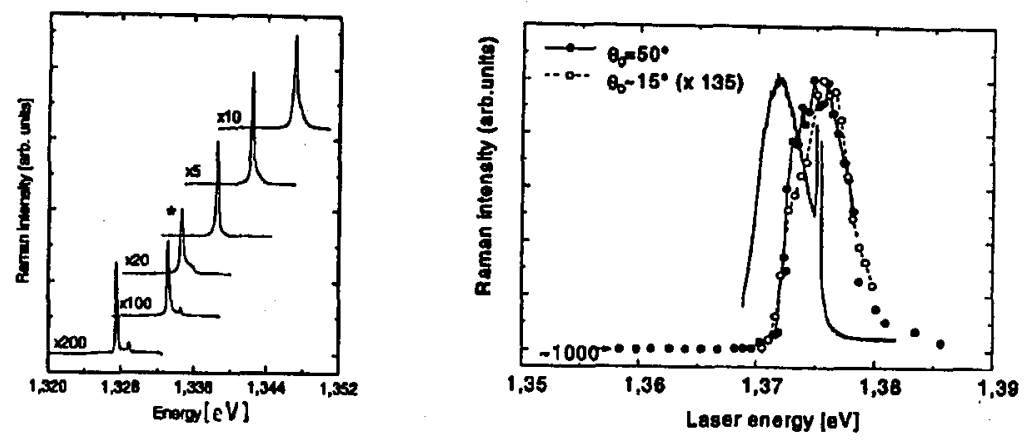

Fig. 5. Variation of the Raman intensity across the exciton energy: typical Raman spectra (left) and comparison between the intensity variations at two different angles and the luminescence line (right).

resonant enhancement is added. We recall that the DOR signal is already several orders of magnitude larger than that of optically unconfined QW's. The magnitude of the observed CPMS resonance indicates that the cavity finesse is not significantly reduced due to absorption at the QW's resonance. In fact, the 250 observed exciton-like resonant enhancement is of the same order as that observed for incoming resonances in multiple quantum wells (MQW's) [8], indicating that, in the microcavity, it adds to the almost unperturbed DOR contribution. The CPMS scan maximum is blue shifted with respect to the pure-exciton luminescence peak (solid curve in Fig. 5). The same is also observed when the resonance scan is taken with a detuned incidence angle, as shown for $\theta_{0}=15^{\circ}$ with open circles in Fig. 5 (note that the signals are weaker and can only be detected in the exciton resonance region). This demonstrates that such a shift is not related with the Rabi splitting. In fact, it is known from resonant Raman scattering in inhomogeneously broadened QW's to be closely related to the emission-absorption "Stokes shift" [8]. More important here is that the CPMS scan of Fig. 5 displays a single maximum, while one would expect a doublet as a signature of the cavity mode anticrossing. The overall energy variation of the Raman cross-section thus does not exhibit any specific signature of the existence of cavity polaritons in the studied sample.

The mediation of cavity-polariton modes as intermediate steps in the scattering process can be however evidenced from the incidence angle dependence of the resonant Raman intensity (see Fig. 4). In Fig. 6 we present the Raman intensity as a function of $\theta_{0}$, recorded with $E_{\mathrm{L}}=E_{x}$ (solid symbols). For comparison, an angular dependence obtained with $E_{\mathrm{L}} \ll E_{x}$, i.e., in the DOR regime, is also shown (open circles). The latter decreases steeply on detuning, and gets below the detection noise level within less than $10^{\circ}$ of the DOR condition. In contrast, for $E_{\mathrm{L}}$ at the exciton resonance the curves decrease much more smoothly and no complete suppression is observed. If cavity and exciton were not coupled, the Raman intensity should fall steeply with $\theta_{0}$ reflecting the cavity finesse as explained in Sec. 2. However, it is clear that a different regime exists for $E_{\mathrm{L}}=E_{x}$.

The cavity-polariton picture provides a natural explanation for the angular dependences in Fig. 6. Though a theory of cavity-polariton mediated scattering 


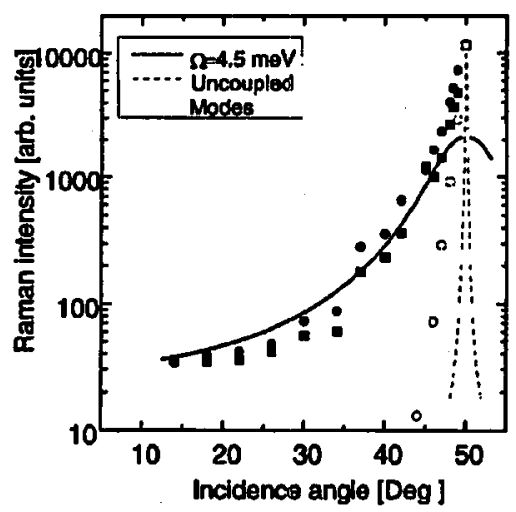

Fig. 6. Incidence angle dependence of the Raman intensity around DOR (open circles) and around CPMS conditions (full dots), respectively, compared to the predictions of the purely photonic model (dashed line) and the one taking into account both excitonic and photonic components of the cavity polariton (full line).

has not yet been developed, we can adapt that originally derived for bulk samples to derive its main consequences regarding our experimental geometry. A polariton mediated Raman event has been described for bulk samples as the transmission of an incoming photon at the interface as a polariton inside the sample $\left(T_{\mathrm{i}}\right)$, followed by scattering from one polariton state $\left(\left|K_{i}\right\rangle\right)$ to another $\left(\left|K_{\mathrm{s}}\right\rangle\right)$ inside the crystal, and subsequent transmission of the scattered polariton outside as a photon $\left(T_{\mathbf{s}}\right)$. The probability of this process has been calculated by first-order perturbation theory as $[6,7]$ :

$$
\sigma_{2} \propto T_{\mathrm{i}} T_{\mathrm{s}}\left|\left\langle K_{\mathrm{i}}\left|H_{\mathrm{e}-\mathrm{ph}}\right| K_{\mathrm{s}}\right\rangle\right|^{2} \rho_{\mathrm{s}} \tau_{\mathrm{l}}
$$

where $\rho_{\mathrm{s}}$ is the density of final polariton states and $r_{1}$ - the lifetime of the initial polariton state before it dephases or leaves the sample. The $T$ 's in Eq. (3) are related in bulk with the nontrivial problem of "additional boundary conditions in dispersive media". In contrast, due to $k_{\|}$conservation in a planar microcavity they are univocally determined. They can be calculated as the cavity-photon transmission factor (which can be defined to reflect the optical enhancement and hence is typically larger than 1), weighted by the polariton photonic strength. In our experimental conditions, the quantities $T_{\mathbf{s}}, \rho_{\mathrm{s}},\left|K_{\mathbf{s}}\right\rangle$, related to the scattered photon remain constant within the whole energy range. As concerns the incident photon, $T_{\mathrm{i}}$ is proportional to the incident photon weight $\sin ^{2} \phi$ while the polariton phonon matrix element is proportional to the incident exciton weight $\cos ^{2} \phi$, because polaritons interact with phonons only through their exciton component. The cavity-polariton lifetime $\tau_{\mathrm{i}}$, on the other hand, has been shown to increase only by a factor approximately 2 going from zero to maximum detuning [9]. Neglecting this latter small contribution, the resonant Raman scattering intensity can be hence simply approximated as

$$
\sigma_{2}\left(\theta_{0}\right) \propto \sin ^{2} \phi \cos ^{2} \phi .
$$

Note that $\Delta$ is related to $\theta_{0}$ by

$$
\Delta=E_{x}-E_{\mathrm{s}} / \cos \left(\theta_{0} / n_{\mathrm{eff}}\right),
$$


where $n_{\text {eff }}$ is the cavity effective refractive index. It is shown with a solid curve in Fig. 6, calculated for $Q=4.5 \mathrm{meV}$, deduced from reflectivity measurements, with the constant prefactor in $\sigma_{2}$ fitted to the Raman intensity at $\theta_{0}=15^{\circ}$. The experiment is well described, except in the region of small detuning. We can understand this discrepancy by arguing that CPMS is accompanied by a weak-coupling contribution which falls as the finesse. These exciton mediated Raman processes contribute, according to Fig. 6 , up to $3 / 5-4 / 5$ of the total CPMS signal, thus providing a possible explanation of the observation of a single peak in the CPMS scan in Fig. 5. In that sense, the angle experiment extracts the cavity-polariton mediated component from the total Raman cross-section.

\section{Conclusion}

To summarize, we have shown that huge enhancements of the Raman cross-sections have been obtained in microcavities, which can be reasonably reproduced quantitatively by a simple combination of the incident field enhancement and the radiation redistribution. We would like to point out the potentialities of the results presented here. On one hand, we have demonstrated the cavity geometry as a promising tool to study the excitations in weakly scattering objects, such as could be single interfaces or small quantities of quantum dots. Moreover, the possibility of selectively amplifying chosen regions of otherwise intense signals enables, for instance, the observation of subtle features in the spectra. On the other hand, it would be very interesting to search for stimulated Raman processes in these structures, which should no doubt present strongly reduced thresholds opening the way to their use in optical devices.

\section{References}

[1] A. Fainstein, B. Jusserand, V. Thierry Mieg, Phys. Rev. Lett. 75, 3764 (1995).

[2] A. Fainstein, B. Jusserand, V. Thierry Mieg, Phys. Rev. Lett. 78, 1576 (1997).

[3] A. Fainstein, B. Jusserand, Phys. Rev. B 54, 11505 (1996).

[4] A. Fainstein, B. Jusserand, V. Thierry Mieg, Phys. Rev. B 53, R13287 (1996).

[5] See, for example, Confined Electrons and Photons: New Physics and Applications, Eds. E. Burstein, C. Weisbuch, Plenum, New York 1995.

[6] C. Weisbuch, R.G. Ulbrich, in: Light Scaltering in Solids, Vol. 3, Eds. M. Cardona, G. Guntherodt, Springer Verlag, Berlin 1982.

[7] B. Bendow, Springer Tracts in Modern Physics 82, 69 (1978).

[8] A.J. Shields, G.O. Smith, E.J. Mayer, R. Eccleston, J. Kuhl, M. Cardona, K. Ploog, Phys. Rev. B 48, 17338 (1993).

[9] B. Sermage, S. Long, I. Abram, J.Y. Marzin, J. Bloch, R. Planel, V. Thierry-Mieg, Phys. Rev. B 53, 16516 (1996). 